\title{
Effects of Cold Agglutinin on the Accuracy of Complete Blood Count Results and Optimal Sample Pretreatment Protocols for Eliminating Such Effects
}

John Hoon Rim, M.D. ${ }^{1,2,3}$, Myung Hee Chang, M.D. ${ }^{4}$, Joowon Oh, M.D. ${ }^{1}$, Heon Yung Gee, M.D. ${ }^{2,3}$, Jeong-Ho Kim, M.D. ${ }^{1}$, and Jongha Yoo, M.D. ${ }^{1,5}$

Department of Laboratory Medicine ${ }^{1}$, Severance Hospital, Yonsei University College of Medicine, Seoul; Department of Pharmacology ${ }^{2}$, Brain Korea 21 PLUS Project for Medical Sciences, Yonsei University College of Medicine, Seoul; Department of Medicine ${ }^{3}$, Physician-Scientist Program, Yonsei University Graduate School of Medicine, Seoul; Departments of Internal Medicine ${ }^{4}$ and Laboratory Medicine ${ }^{5}$, National Health Insurance Service Ilsan Hospital, Goyang, Korea

\section{Dear Editor,}

Cold agglutinin is an autoantibody that causes autoimmune hemolytic anemia by binding to $1 / i$ carbohydrate antigens on the red blood cell (RBC) surface [1]. While RBC agglutination causes clinical symptoms of hemolytic anemia, agglutination caused by cold agglutinin is a notorious pre-analytical and analytical factor that leads to spurious automated complete blood count (CBC) results [2]. Although RBC agglutination falsely increases mean corpuscular volume (MCV), effects of cold agglutinin on other CBC parameters have not been widely studied $[3,4]$. The effects of cold agglutinin on $\mathrm{CBC}$ results have been evaluated mainly for a limited number of parameters, on old versions of automated CBC analyzers [5, 6], which have undergone remarkable technical improvements in the last decade. We present a case of chronic cold agglutinin disease, and report the effects of cold agglutinin on CBC parameters based on whole blood samples comparatively analyzed by four automated CBC analyzers commonly used in current clinical laboratories.

A 56-year old male patient visited our hospital for low hemoglobin $(\mathrm{Hb})$ concentration, fatigue, and hematuria. The initial $\mathrm{CBC}$ results revealed the following: $\mathrm{Hb}, 102 \mathrm{~g} / \mathrm{L}$; platelets, 275 $\times 10^{9} / \mathrm{L}$; and white blood cell (WBC) count, $4.70 \times 10^{9} / \mathrm{L}$. Peripheral blood smear showed normocytic normochromic anemia with mild anisopoikilocytosis, mild elliptocytosis, and dacryocytes. RBC clumps that resolved after sample incubation at $37^{\circ} \mathrm{C}$ suggested the diagnosis of cold agglutinin disease. The patient required no specific treatment because symptoms were not severe. Followup seasonal variations in symptoms and $\mathrm{CBC}$ results were observed. The institutional review board of National Health Insurance Service Ilsan Hospital approved this study (IRB number: NHIMC 2015-03-011).

In total, $16 \mathrm{~K}_{2}$ EDTA samples of whole blood were collected. The first sample was analyzed on the XE-2100 (Sysmex, Kobe, Japan) immediately after collection, the results of which served as reference values. The four most commonly used automated CBC analyzers-XE-2100, XN-1000 (Sysmex), ADVIA 2120i (Siemens Diagnostics, Tarrytown, NY), and Unicel DxH 800 (Beckman Coulter Inc., Fullerton, CA)—were used for comparative analysis. The experimental flowchart is shown in Fig. 1A.

Each automated CBC analyzer measured four samples. System accuracy was compared using the unit of delta percentage difference (DPD) from the reference value, which was defined
Received: July 26, 2017

Revision received: October 22, 2017

Accepted: February 27, 2018

Corresponding author: Jongha Yoo

Department of Laboratory Medicine, National Health Insurance Service IIsan Hospital, 100 Ilssan-ro, Ilsandong-gu, Goyang 10444, Korea

Tel: +82-31-900-0909, Fax: +82-31-900-0925

E-mail: jhyooken@gmail.com

\section{(C) Korean Society for Laboratory Medicine}

This is an Open Access article distributed under the terms of the Creative Commons Attribution Non-Commercial License (http://creativecommons.org/licenses/by-nc/4.0) which permits unrestricted non-commercial use, distribution, and reproduction in any medium, provided the original work is properly cited. 
according to a previous report [7] as follows:

Delta Percentage $=\frac{(\text { Test analyzer test tube }- \text { reference analyzer reference tube })}{} \times 100$ Difference $(\%)=\frac{\text { Reference analyzer reference tube }}{2}$

We analyzed the effects of analyzer type, storage temperature, storage duration, and incubation period, using eight $\mathrm{CBC}$ parameters [RBC count, $\mathrm{Hb}$, hematocrit, $\mathrm{MCV}$, mean corpuscular hemoglobin $(\mathrm{MCH})$, mean corpuscular hemoglobin concentration (MCHC), WBC count, and platelet count] in terms of DPD. We interpreted the results as positive or negative bias, or acceptable. The criteria for spurious effects were derived from three guidelines for quality requirements for automated CBC analyzers (Table 1) [8-10].

When we compared three temperature conditions [i.e., measurements (1) or (2), (3), and (4) in Fig. 1A, according to analyzer], the sample stored for 1 hour at $37^{\circ} \mathrm{C}$ showed the smallest deviance from the reference value based on the DPD value (Fig. $1 \mathrm{~B}$, Table 1). Interestingly, $\mathrm{Hb}$ concentration and platelet count were not highly affected by storage temperature, while WBC count showed variability according to the analyzer used. For all analyzers, except the XN-1000, sample storage at $37^{\circ} \mathrm{C}$ for 1 hour yielded more accurate results than storage at $37^{\circ} \mathrm{C}$ for 24 hours. When we compared samples stored at $37^{\circ} \mathrm{C}$ for 1 hour with samples stored at $4^{\circ} \mathrm{C}$ for 1 hour followed by incubation at $37^{\circ} \mathrm{C}$ for 1 hour, there was no significant difference in any of the parameter values (Fig. 1C). Based on these results, we propose two practically optimal protocols: (1) measuring CBC within 1 hour if the sample is stored at $37^{\circ} \mathrm{C}$ after collection and (2) measuring $\mathrm{CBC}$ after incubation at $37^{\circ} \mathrm{C}$ for 1 hour if the sample is stored at $4^{\circ} \mathrm{C}$ for a short term.

An interesting finding of our study was that $\mathrm{Hb}$ concentration showed the smallest variance among all parameters evaluated in this study. This finding is consistent with those of previous studies [2,3] and is likely owing to the fact that we measured $\mathrm{Hb}$ after RBC lysis, which eliminates clumping by cold agglutinin. From a clinical aspect, it is reassuring that the most important variable for determining the need for RBC transfusion is not substantially distorted by the presence of cold agglutinin.

Thus, we demonstrated spurious effects of cold agglutinin on eight parameters comparatively measured on four automated $\mathrm{CBC}$ analyzers. Our results indicated that cold agglutinin affects not only MCV but also other parameters, depending on analyzer type. Optimal pretreatment protocols to eliminate spurious effects of cold agglutinin on results generated by automated CBC analyzers are needed.

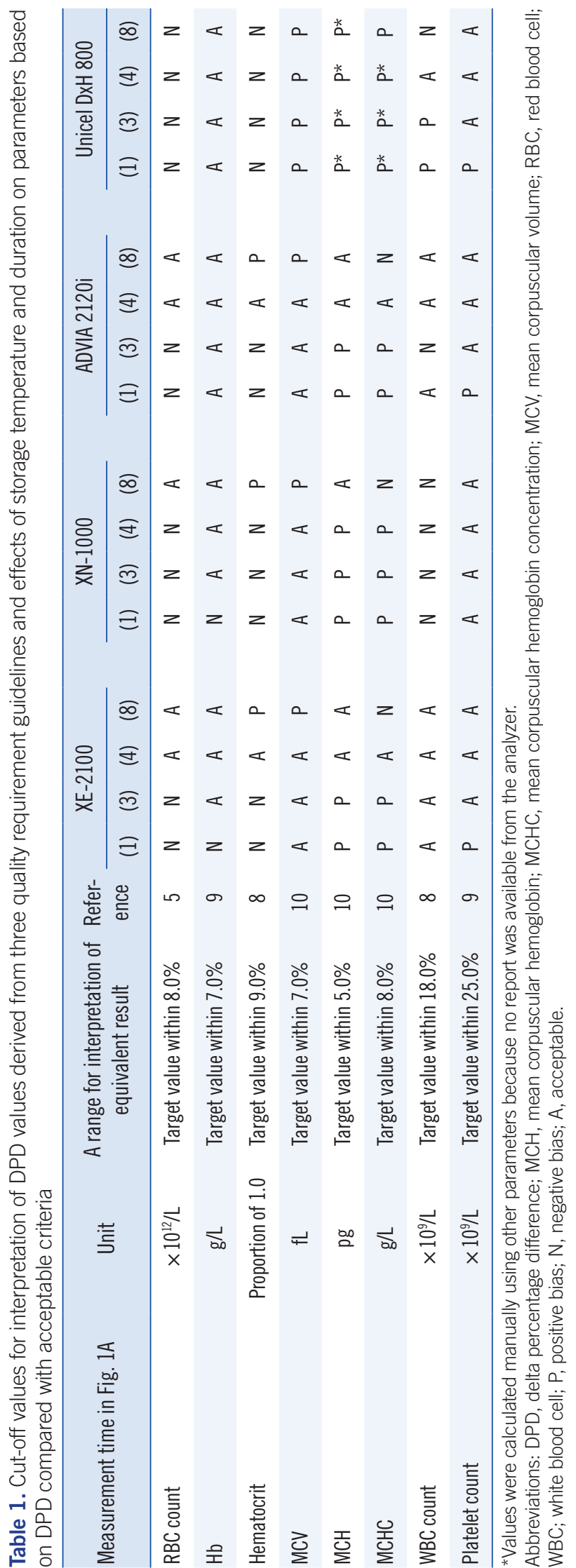


Rim JH, et al.

Cold agglutinin effects on $\mathrm{CBC}$ results
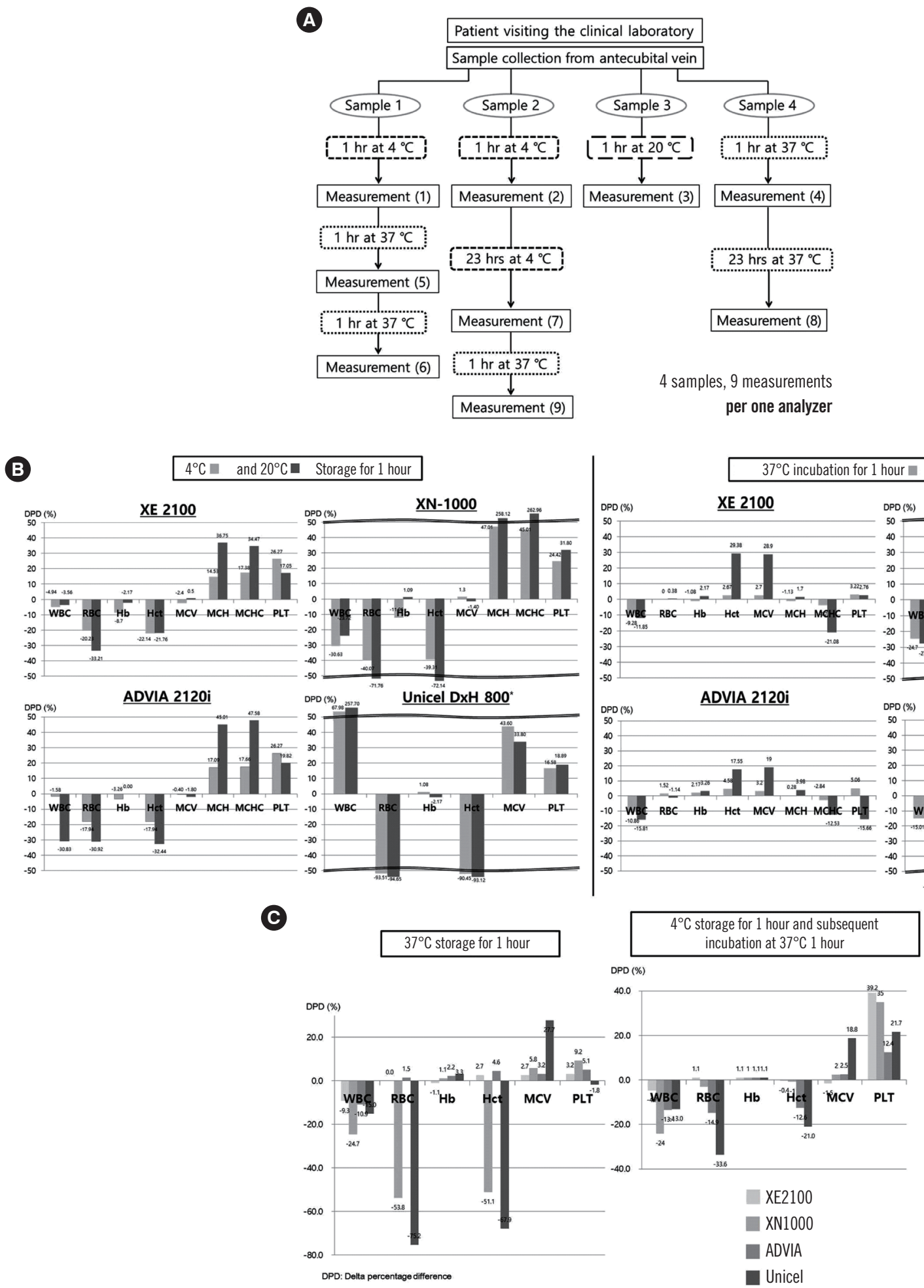

Fig. 1. Summary of study design and comparative assay results. (A) Experimental flowchart of sample collection and measurements. In total, four samples were evaluated in nine measurements per analyzer. Thus, 16 samples in total were evaluated 36 times, on four different automated CBC analyzers. (B) Effects of storage temperature and duration on CBC parameters measured with the four analyzers. (C) Comparison of the four automated $\mathrm{CBC}$ analyzers applying two optimal protocols, which were (1) measuring CBC within 1 hour of storage at $37^{\circ} \mathrm{C}$ and (2) measuring $\mathrm{CBC}$ after short-term storage at $4^{\circ} \mathrm{C}$ for 1 hour and subsequent incubation at $37^{\circ} \mathrm{C}$ for 1 hour. 


\section{Authors' Disclosures of Potential Conflicts of Interest}

No potential conflicts of interest relevant to this article were reported.

\section{REFERENCES}

1. Swiecicki PL, Hegerova LT, Gertz MA. Cold agglutinin disease. Blood 2013;122:1114-21.

2. Zandecki M, Genevieve F, Gerard J, Godon A. Spurious counts and spurious results on haematology analysers: a review. Part II: white blood cells, red blood cells, haemoglobin, red cell indices and reticulocytes. Int J Lab Hematol 2007;29:21-41.

3. Ercan S, Caliskan M, Koptur E. 70-year old female patient with mismatch between hematocrit and hemoglobin values: the effects of cold agglutinin on complete blood count. Biochem Med 2014;24:391-5.

4. Gulati G, Song J, Florea AD, Gong J. Purpose and criteria for blood smear scan, blood smear examination, and blood smear review. Ann Lab Med 2013;33:1-7.

5. Medicare, Medicaid and CLIA programs; regulations implementing the Clinical Laboratory Improvement Amendments of 1988 (CLIA)--HCFA. Final rule with comment period. Fed Regist 1992;57:7002-186.

6. Ricos C, Ramon F, Salas A, Buno A, Calafell R, Morancho J, et al. Minimum analytical quality specifications of inter-laboratory comparisons: agreement among Spanish EQAP organizers. Clin Chem Lab Med 2012; 50:455-61.

7. Park YJ, Rim JH, Yim J, Lee SG, Kim JH. Effects of two types of medical contrast media on routine chemistry results by three automated chemistry analyzers. Clin Biochem 2017;50:719-25.

8. Bessman JD and Banks D. Spurious macrocytosis, a common clue to erythrocyte cold agglutinins. Am J Clin Pathol 1980;74:797-800.

9. Solanki DL and Blackburn BC. Spurious red blood cell parameters due to serum cold agglutinins: observations on Ortho ELT-8 cell counter. Am J Clin Pathol 1985;83:218-22.

10. Hattersley PG, Gerard PW, Caggiano V, Nash DR. Erroneous values on the Model $S$ Coulter Counter due to high titer cold autoagglutinins. Am J Clin Pathol 1971;55:442-6. 